\title{
Resiliência: Uma Análise a Partir das Características Sociodemográficas da População Brasileira
}

\author{
Cynthia de Freitas Melo ${ }^{1}$ \\ José Eurico de Vasconcelos Filho ${ }^{1}$ \\ Marina Braga Teófilo ${ }^{1}$ \\ Amanda Martins Suliano ${ }^{1}$ \\ Érika Carolinne Cisne \\ Ronaldo Almeida de Freitas Filho ${ }^{T}$ \\ ${ }^{1}$ Universidade de Fortaleza
}

\begin{abstract}
Resumo
Objetivou-se analisar o efeito de dados sociodemográficos sobre o nível de resiliência de uma parcela da população brasileira. Para tanto, contou-se com uma amostra nacional não probabilística composta por 2.038 participantes, que responderam à Escala de Resiliência desenvolvida por Wagnild e Young. Os dados foram analisados por meio de estatística descritiva e bivarida com auxílio do software SPSS (Statistical Package for Social Science). Entre os resultados, verificou-se uma média geral baixa nos índices de resiliência $(M=124,60 ; D P=22,69)$. Constatou-se também indicativos de que pessoas com determinadas características sociodemográficas têm maior tendência à resiliência: pessoas com religião, com maior nível de escolaridade, divorciados/ casados, aposentados, sem piercing, não fumante, sem histórico de desistência ou repetição em anos escolares. Conclui-se que tais características podem estar relacionadas à capacidade da pessoa ser resiliente, mas não ser determinante, no nível de resiliência. Essas informações são primordiais para compreender os fatores que predispõem à resiliência da população. Palavras-chave: resiliência, indicadores de resiliência, desenvolvimento de resiliência
\end{abstract}

\section{Resilience: An Analysis based on the sociodemographic characteristics of the Brazilian population}

\begin{abstract}
The objective of this study was to analyze the effect of sociodemographic data on the level of resilience of a portion of the Brazilian population. For this purpose, a non-probabilistic national sample composed of 2,038 participants answered the Resilience Scale developed by Wagnild and Young. Data were analyzed using descriptive and bivariate statistics with the help of SPSS (Statistical Package for Social Sciences) software. Among the results, there was a low overall mean in resilience indices (M $=124.60, \mathrm{SD}=22.69)$. There were also indications that people with certain sociodemographic characteristics tend to be more resilient: people who are religious, with a higher education level, divorced / married, retired, without piercing, non- smoker, without a history of dropout or repetition in school years. It can be concluded that such characteristics may be related to a person's ability to be resilient, but not determinant, in the level of resilience. This information is essential to understand the factors that predispose the population to resilience.

Keywords: resilience; indicators of resilience; development of resilience.
\end{abstract}

Resiliencia: Análisis de las Características Sociodemográficas de la Población Brasileña

\section{Resumen}

El objetivo de este estudio fue analizar el efecto de datos sociodemográficos sobre el nivel de resiliencia de una parte de la población brasileña. Para ello, se contó con una muestra nacional no probabilística compuesta por 2.038 participantes, que respondieron la Escala de Resiliencia desarrollada por Wagnild y Young. Los datos fueron analizados por medio de estadística descriptiva y bivariada con ayuda del software SPSS (Statistical Package for Social Science). Entre los resultados, se constató un promedio general bajo en los índices de resiliencia $(M=124,60, \mathrm{DP}=22,69)$. Se verificaron también indicativos de que personas con determinadas características sociodemográficas tienen mayor tendencia a resiliencia: personas con religión, con mayor nivel de escolaridad, divorciados / casados, jubilados, sin piercing, no fumadores, sin histórico de desistencia o repetición de años escolares. Se concluye que tales características pueden estar relacionadas con la capacidad de la persona de ser resistente, pero no ser determinante, a nivel de resiliencia. Estas informaciones son primordiales para comprender los factores que predisponen la resiliencia de la población.

Palabras clave: resiliencia; indicadores de resiliencia; desarrollo de resiliencia

Entre as décadas de 1970 e 1980, intensificaram-se os estudos direcionados à compreensão de pessoas que, apesar de expostas a severas adversidades, como pobreza, riscos biológicos e instabilidade familiar, permaneciam saudáveis e desprovidas de comorbidades psiquiátricas (Rutter, 1985; Werner \& Smith, 1977; 1982; 1992). Inicialmente, essas pessoas foram chamadas de invulneráveis, pois eram indivíduos que, além de não se debilitarem diante das dificuldades, ainda demonstravam ter recursos para o próprio aperfeiçoamento, tanto no 
âmbito pessoal quanto no social. Ante questionamentos sobre o fenômeno, os pesquisadores perceberam haver indivíduos que reagem melhor em situações de desafio, enquanto outros conseguem enfrentar determinadas circunstâncias diante do modelo de danos (Munist et al., 1998), fato que mais tarde seria nomeado como resiliência (Laranjeira, 2007).

Desde então, a relevância do estudo sobre a resiliência cresce devido ao fato de esse assunto possibilitar um reconhecimento dos aspectos de risco, além de proporcionar a procura por aptidões pessoais e circunstanciais que podem ser utilizadas para encarar situações de instabilidade. A partir do aspecto social, a pesquisa acerca da resiliência demonstra uma nova perspectiva sobre como uma grande parcela social, em situação de risco, consegue lidar com as questões adversas vivenciadas em seu cotidiano (Fontes, Fattori, D’Elboux, \& Guariento, 2015), uma vez que se entende que a resiliência pode ser aprendida a partir das experiências enfrentadas nesse contexto.

Nesse sentido, o conceito de resiliência é compreendido como uma capacidade ou até mesmo uma habilidade humana de adequar-se às circunstâncias de crise nos âmbitos psicológico e biológico, aprimorando-se habilidades intrapsíquicas; vinculadas ao meio afetivo e social e permitindo associar-se uma formulação psíquica congruente à inserção social. Assim, as pessoas podem utilizar-se de uma pluralidade de respostas e de comportamentos adaptativos para resoluções de ações e valores em contexto de adversidade (Laranjeira, 2007).

Considera-se que algumas pessoas podem apresentar conflitos familiares ou sociais que não podem ser ignorados, enquanto outras, em situações semelhantes de adversidade, não demonstram alterações ou desajustes psíquicos. Diante disso, surge a definição de resiliência como um potencial de renovar-se e fortificar-se em meio a situações traumáticas, implicando na habituação e no reajuste em meio ao infortúnio, em constante manutenção de práticas direcionadas para a autoconfiança, resolução de problemas e adaptação ao contexto (McCann et al., 2013).

Para haver condições de enfrentamento de uma determinada situação traumática e, consequentemente, a capacidade de um novo desenvolvimento, ressalte-se, podem existir, muitas vezes, duas opções. Uma delas é a pessoa passar a viver em constante tensão, pautada comumente na proteção contra as agressões do mundo e baseada em mecanismos de adaptação às dores físicas e psíquicas. A outra é a pessoa encontrar condições que são favorecidas pela forma como o ambiente reconhece e trata o seu trauma, deparando-se com outros investimentos na sua rotina e criando novas oportunidades de viver, podendo identificar, inclusive, a capacidade de resolução de problemas de forma independente e determinada (Coimbra \& Morais, 2015).

A saída do fato gerador de estresse ou evento traumático pode ser pensada como uma possibilidade de desencadear movimentos eficazes; contra uma sequência de repetições de atitudes paralisadas, estabelecida pelo trauma e que pode permitir que a pessoa se desvencilhe do sofrimento e isolamento acometidos. Além disso, tal saída pode ser capaz de integrar o indivíduo ao seu meio social e favorecer a resiliência (Coimbra \& Morais, 2015; Laranjeira, 2007).

Ao levar em consideração os períodos durante a formação e o desenvolvimento humano, torna-se fundamental a compreensão de que, nos estágios em que o organismo está sensível à aprendizagem, são construídas relações que estabelecem vínculos, proporcionando a constituição dos recursos internos. É por meio dessas experiências que se aprende a forma de relacionamento com o mundo, bem como as diversas maneiras de enfrentamentos e reações para solucionar problemas, favorecendo a vinculação segura e confiança básica que possibilitarão a construção da resiliência (Andrade, 2011; Haack, Vasconcellos, Pinheiro, \& Prati, 2012; Motta \& Rosa, 2016; Oliveira \& Godoy, 2015).

Diante disso, verificou-se que algumas condições são necessárias para uma possível retomada de uma situação de vida semelhante à anterior ao fato gerador de estresse. Esta pesquisa pretende, assim, investigar a resiliência na população brasileira e sua relação com determinadas características sociodemográficas, uma vez que a literatura revela estarem correlacionadas algumas dessas a uma maior ou menor resiliência (Coimbra \& Morais, 2015; Sousa \& Araújo, 2015).

A fim de compreender o nível de desenvolvimento científico sobre o tema, observaram-se os resultados de algumas pesquisas, a seguir descritas, que abordaram isoladamente a relação de algumas características das pessoas com o seu nível de resiliência, a destacar gênero, religiosidade/espiritualidade e vulnerabilidade social (Ferreira, 2013; Haack et al., 2012; Melo, Sampaio, Souza, \& Pinto, 2015; Mosqueiro, 2015; Ripar, Evangelista, \& Paula, 2008; Silva, Pinto, Nogueira, \& Ferreira, 2014; Soratto, Silva, Zugno, \& Daniel, 2016). Um trabalho realizado com discentes de administração, com o objetivo de comparar a resiliência entre os sexos, 
apresentou como resultado a constatação de mínima diferença entre homens e mulheres quanto aos recursos de enfrentamento das situações adversas, concluindo uma similaridade da capacidade de enfrentamento entre os sexos (Silva et al., 2014). Outra pesquisa realizada, com o objetivo de investigar o perfil de resiliência mais específico entre o gênero masculino e feminino de 27 adolescentes do ensino médio da cidade de São Bernardo do Campo (SP), mostrou que ambos os sexos se assemelham nos fatores otimismo para a vida e controle dos impulsos e apresentam diferenças significativas nos fatores autoeficácia e possibilidade de alcançar pessoas. Concluíram que alguns aspectos da resiliência não dependem do gênero, mas que, de alguma forma, as pessoas selecionam estratégias para enfrentar e solucionar problemas de acordo com suas características de gênero (Ripar et al., 2008).

No que diz à relação entre resiliência e religiosidade, uma pesquisa realizada com 143 pacientes com depressão internados em um hospital de Porto Alegre detectou que a resiliência se apresenta associada ao índice de resiliência e bem-estar nesses sujeitos (Mosqueiro, 2015). De modo semelhante, uma pesquisa com 60 pacientes oncológicos de hospitais em São Paulo mostrou que a presença da espiritualidade é um fator de influência na capacidade de resiliência para enfrentar o processo de adoecimento direcionado para o tratamento oncológico (Soratto et al., 2016). Desse modo, pode-se concluir que pessoas com espiritualidade/religiosidade possuem melhores mecanismos de enfrentamento do que agnósticos (Melo et al., 2015).

Também é possível relacionar o índice de resiliência às famílias que vivem em situação de vulnerabilidade social. Em pesquisa com 10 famílias que participam do Programa Bolsa Família no município de Sumé - PB, mostrou-se que estas passaram a ser mais resilientes, ou seja, confiantes na superação de seus problemas sociais diante da contribuição do programa (Ferreira, 2013). Ainda, outro estudo realizado com 35 adolescentes em vulnerabilidade social indicou que alguns jovens que participavam de programas de intervenção social-comunitária apresentaram melhor resiliência (Haack et al., 2012). Concluíram, portanto, que as adversidades dificultam a emissão de respostas satisfatórias e o desenvolvimento de habilidades.

A literatura sinaliza ainda que, como consequência das limitações na capacidade de resiliência, algumas pessoas podem recorrer a outros mecanismos para extravasar seus sentimentos, como uso de piercing ou tatuagem (Teixeira, 2012), fumo (Fortes, Portuguez, \&
Argimon, 2009), medicação psiquiátrica ou drogas (Oliveira, Siqueira, Santos, \& Furegato, 2014). Podem ainda apresentar reações como reprovação ou evasão na escola ou universidade (Cookson, 1990; Duarte, 2016; Lopes \& Massinelli, 2013). Tais circunstâncias podem sinalizar, mas não determinar, deficiência de resiliência e presença de comportamentos de risco.

Portanto, vale ressaltar que os aspectos relacionados à resiliência são possíveis de serem aprendidos durante a história de vida das pessoas e a depender do meio social em que convivem, sendo fundamental o conhecimento sobre as condições que favorecem um maior ou menor índice de resiliência. Ademais, atenta-se para o fato de que alguns dados sociodemográficos não foram possíveis de serem verificados nas pesquisas citadas, podendo indicar uma escassez de aprofundamento dos estudos nesse âmbito.

Frente aos dados preliminares encontrados em estudos recentes sobre a relação entre características pessoais e o nível de resiliência, a presente pesquisa teve como objetivo analisar o efeito de características sociodemográficas sobre o nível de resiliência de uma parcela da população brasileira, visando, assim, a colaborar com a literatura científica e com a oferta de informações a serem utilizadas na construção de resiliência e nas estratégias de promoção da qualidade de vida e amparo nas questões referentes ao adoecimento.

\section{Método}

\section{Tipo de Pesquisa}

Para avaliar o efeito das características sociodemográficas sobre o nível de resiliência de uma parcela da população brasileira, foi realizada uma pesquisa descritiva e exploratória, com levantamento via internet em todo o Brasil. Por meio desse tipo de pesquisa, pode-se descrever o fenômeno a partir de questionamento direto, abrangendo-se uma amplitude de participantes.

\section{Amostra de Participantes}

A população do Brasil é de 198,7 milhões de habitantes. Para uma população desse porte, com nível de confiança de $95 \%$ e erro amostral de 5\%, fez-se necessário uma amostra mínima de 385 participantes. Esses foram selecionados por meio de uma amostra não probabilística por conveniência (Favero \& Belfiore, 2017). Após a coleta de dados, contou-se com 2.038 participantes, com média de idade de 27,07 ( $D P=29,91)$, cujos dados sociodemográficos podem ser visualizados na Tabela 1. 
Entre as características de inadaptação, detectou-se que uma parcela significativa deu início a cursos universitários que não foram concluídos $(f=893 ; 43,80 \%)$, repetiu série do ensino fundamental ou médio $(f=363$;
$17,80 \%)$, repetiu disciplina do ensino superior $(f=849$; $41,70 \%$ ), mudou de emprego por motivo de briga ou desentendimento $(f=362 ; 17,80 \%)$, mudou de cidade por insatisfação com a vida local $(f=436 ; 21,40 \%)$ e

Tabela 1.

Dados Sociodemográficos da Amostra

\begin{tabular}{|c|c|c|c|}
\hline \multirow{2}{*}{\multicolumn{2}{|c|}{$\begin{array}{c}\text { Variável } \\
f\end{array}$}} & \multicolumn{2}{|c|}{ Distribuição } \\
\hline & & $\%$ & \\
\hline \multirow{2}{*}{ Sexo } & Masculino & 609 & $29,90 \%$ \\
\hline & Feminino & 1429 & $70,10 \%$ \\
\hline \multirow{4}{*}{ Estado civil } & Solteiro & 1.301 & $63,80 \%$ \\
\hline & Casado & 363 & $17,08 \%$ \\
\hline & União estável & 184 & $9,00 \%$ \\
\hline & Outros & 190 & $9,30 \%$ \\
\hline \multirow{4}{*}{ Religião } & Católicos & 809 & $39,70 \%$ \\
\hline & Agnósticos & 396 & $19,40 \%$ \\
\hline & Cristão Protestante & 301 & $14,80 \%$ \\
\hline & Outras & 532 & $26,10 \%$ \\
\hline \multirow{4}{*}{ Escolaridade } & Ensino médio & 205 & $10,10 \%$ \\
\hline & Ensino Superior & 911 & $44,70 \%$ \\
\hline & Ensino superior & 388 & $19 \%$ \\
\hline & Pós-graduação & 482 & $23,70 \%$ \\
\hline \multirow{4}{*}{ Trabalho } & Empregado & 877 & $43,00 \%$ \\
\hline & Desempregado & 591 & $29,00 \%$ \\
\hline & Profissional & 179 & $8,80 \%$ \\
\hline & Outros & 392 & $19,20 \%$ \\
\hline \multirow{4}{*}{ Renda mensal } & Menos de 1.000 & 650 & $31,90 \%$ \\
\hline & De 1.000 a 2.999 & 611 & $30,00 \%$ \\
\hline & Outros valores & 539 & $26,40 \%$ \\
\hline & Não sabe/quer informar & 238 & $11,70 \%$ \\
\hline \multirow{2}{*}{ Adoção } & Sim & 73 & $3,60 \%$ \\
\hline & Não & 1965 & $96,40 \%$ \\
\hline \multirow{4}{*}{ Tatuagem } & Nenhuma & 1609 & $78,90 \%$ \\
\hline & Uma & 196 & $9,60 \%$ \\
\hline & Duas & 81 & $4,00 \%$ \\
\hline & Três ou mais & 152 & $7,40 \%$ \\
\hline \multirow[t]{4}{*}{ Piercing } & Nenhum & 1891 & $92,80 \%$ \\
\hline & Uma & 99 & $4,90 \%$ \\
\hline & Duas & 36 & $1,80 \%$ \\
\hline & Três ou mais & 12 & $0,50 \%$ \\
\hline \multirow[t]{2}{*}{ Fumo (últimos 12 meses) } & Sim & 167 & 8,20 \\
\hline & Não & 1871 & $91,80 \%$ \\
\hline
\end{tabular}


toma ou já tomou medicação para transtornos mentais ou neurológicos $(f=523 ; 25,70 \%)$ (ver Tabela 2$)$.

\section{Instrumentos}

Questionário Sociodemográfico. Foi utilizado um questionário sociodemográfico, para caracterização da amostra e comparação entre grupos. Abordou-se informações sobre sexo, filiação, uso de tatuagem e piercing, estado civil, religião, escolaridade, renda e ocupação profissional.

Escala de Resiliência. Utilizou-se a Escala de Resiliência desenvolvida por Wagnild e Young (1993). Tal instrumento é considerado um dos poucos usados para medir níveis de adaptação psicossocial positiva em face de eventos de vida importantes. Optou-se por usar a versão brasileira com adaptação transcultural de
Pesce et al., (2005), que apresentou bons resultados na equivalência semântica dos itens e consistência interna, com alpha de Cronbach 0,80. Possui 25 itens com resposta do tipo Likert, variando de 1 (discordo totalmente) a 7 (concordo totalmente). Os escores da escala oscilam de 25 a 175 pontos, com valores altos indicando elevada resiliência. É composta por três fatores:

- Fator 1 - Resolução de ações e valores, com 14 itens $(1,2,6,8,10,12,14,16,18,19,21,23,24 \mathrm{e}$ 25). Indicam resoluções de ações (levar os planos até o fim; lidar com problemas de alguma forma; aceitar os fatos sem muita preocupação; ser disciplinado; fazer as coisas um dia de cada vez; ser uma pessoa com quem se pode contar em uma emergência; geralmente encarar uma situação de

Tabela 2.

Características de Inadaptação da Amostra

\begin{tabular}{|c|c|c|c|}
\hline \multirow{2}{*}{\multicolumn{2}{|c|}{ Variável }} & \multicolumn{2}{|c|}{ Distribuição } \\
\hline & & $f$ & $\%$ \\
\hline \multirow{4}{*}{ Não concluiu curso superior } & Nenhuma vez & 1145 & $56,20 \%$ \\
\hline & $1 \mathrm{vez}$ & 544 & $26,70 \%$ \\
\hline & 2 vez & 236 & $11,60 \%$ \\
\hline & Mais de 2 vezes & 113 & $5,00 \%$ \\
\hline \multirow{4}{*}{ Repetição de série escolar } & Nenhuma vez & 1675 & $82,20 \%$ \\
\hline & $1 \mathrm{vez}$ & 257 & $12,60 \%$ \\
\hline & 2 vez & 79 & $3,90 \%$ \\
\hline & Mais de 2 vezes & 27 & $1,30 \%$ \\
\hline \multirow{4}{*}{ Reprovação no curso superior } & Nenhuma vez & 1189 & $58,30 \%$ \\
\hline & $1 \mathrm{vez}$ & 361 & $17,70 \%$ \\
\hline & 2 vez & 276 & $13,50 \%$ \\
\hline & Mais de 2 vezes & 212 & $10,40 \%$ \\
\hline \multirow{4}{*}{ Mudança de emprego } & Nenhuma vez & 1676 & $82,20 \%$ \\
\hline & $1 \mathrm{vez}$ & 235 & $11,50 \%$ \\
\hline & 2 vez & 79 & $3,90 \%$ \\
\hline & Mais de 2 vezes & 48 & $3,30 \%$ \\
\hline \multirow{4}{*}{ Mudança de cidade } & Nenhuma vez & 1602 & $78,60 \%$ \\
\hline & $1 \mathrm{vez}$ & 309 & $15,20 \%$ \\
\hline & 2 vez & 80 & $3,90 \%$ \\
\hline & Mais de 2 vezes & 47 & $2,30 \%$ \\
\hline \multirow{3}{*}{ Uso de medicação psiquiátrica } & Não usa & 1515 & $74,30 \%$ \\
\hline & Já usou, mas não usa mais. & 346 & $17,00 \%$ \\
\hline & Usa & 177 & $8,70 \%$ \\
\hline
\end{tabular}


diversas maneiras; normalmente encontrar uma saída quando está em uma situação difícil; ter energia suficiente para fazer o que deve ser feito) e valores (sentir orgulho de ter realizado metas em sua vida; ser amigo de si mesmo; frequentemente encontro motivos para rir; perceber sentido em sua vida e levar em conta o apoio dos valores que dão sentido à vida, como a amizade, a realização pessoal, a satisfação e o significado da vida);

- Fator 2 - Independência e determinação, com seis itens $(5,7,9,11,13$ e 22). Indicam manutenção de interesse pelas coisas, poder estar por sua própria conta, sentir-se bem ainda que haja pessoas que não gostam dele e ser determinado;

- Fator 3 - Autoconfiança e capacidade de adaptação às situações, com cinco itens $(3,4,15,17,20)$. Indicam capacidades como ser capaz de depender de si mais do que de qualquer outra pessoa, sentir que pode lidar com várias situações ao mesmo tempo, pode enfrentar tempos difíceis porque já experimentou dificuldades antes, crer em si mesmo a ponto de sentir-se apto a atravessar tempos difíceis, não insistir em situações sobre as quais não pode fazer nada.

\section{Procedimentos}

Considerando-se os aspectos éticos referentes a pesquisas envolvendo seres humanos, o presente estudo foi submetido à Comissão de Ética em Pesquisa da (Informação retirada pela revista), aprovado com parecer $\mathrm{N}^{\mathrm{o}}$ 1.058.252. Em seguida, o instrumento foi disponibilizado na internet juntamente com o Termo de Consentimento Livre Esclarecido - TCLE, por meio de uma página específica e de domínio privado. A divulgação ocorreu durante quatro meses (novembro a fevereiro/2015), por meio de redes sociais, reportagens televisionadas, revistas e portais digitais. Destaca-se ainda que foram respeitados os aspectos éticos exigidos pela Resolução no 466/12 do Conselho Nacional de Saúde.

\section{Análise dos Dados}

As análises de dados foram realizadas com auxílio do pacote estatístico SPSS (Statistical Package for Social Science) para Windows, versão 22, e divididas em três etapas.

Primeiro, foi traçado o perfil da amostra, por meio de estatística descritiva (frequência, porcentagem e medidas de tendência central e dispersão).
$\mathrm{Na}$ segunda etapa, foi realizada a análise descritiva dos resultados da escala de resiliência. Para tanto, empreendeu-se o somatório das pontuações da escala total de resiliência e de cada fator da escala; para verificação da média de pontuação da população. $\mathrm{Na}$ sequência, verificou-se a quantidade de participantes que tiveram pontuações iguais ou acima da média dos escores (considerando o desvio padrão) e os que ficaram abaixo da média. Ressalte-se que, dessa forma, segue-se a orientação de que não se pode medir resiliência, mas sim verificar a tendência de uma pessoa a ser mais ou menos resiliente do que outras, sinalizando o seu potencial para resiliência, como lembram Poletto, Wagner e Koller (2004).

Ademais, ainda na última etapa das análises, foram realizadas comparações das diferenças entre os grupos nas avaliações dos fatores em função dos dados sociodemográficos: sexo, estado civil, religião, escolaridade, trabalho, renda, adoção, tatuagem, piercing e fumo. Também foram feitas comparações por características de inadaptação: desistência de curso universitário, repetição de série do ensino fundamental/médio e de disciplina no ensino superior, mudança de emprego ou de cidade e uso de medicação psíquica. Destaca-se que todas as variáveis se apresentaram não normais a partir do teste de Shapiro-Wilk: pontuação total da escala ( $W$ $=0,98 ; p<0,01)$, fator $1(W=0,97 ; p<0,01)$, fator $2(W=0,99 ; p<0,01)$, fator $3(W=0,97 ; p<0,01)$. Portanto, foram utilizados os testes não paramétricos Mann-Whitney e Kruskal-Wallis.

\section{Resultados}

A presente pesquisa objetivou analisar a resiliência da população brasileira, verificando se as características sociodemográficas têm efeito sobre o nível de resiliência das pessoas. Nesse sentido, serão apresentados nesta seção, os resultados encontrados na pontuação total de resiliência e nos fatores anteriormente descritos: Fator 1 - Resolução de ações e valores; Fator 2 - Independência e determinação e Fator 3 - Autoconfiança e capacidade de adaptação às situações. Em seguida, também serão apresentados os resultados das comparações por dados sociodemográficos.

A pontuação de Resiliência Total da amostra apresentou uma média de 124,60 (DP $=22,69)$, com pontuação que varia entre 26,00 e 178,00. Observou-se que $906(44,60 \%)$ pessoas apresentaram pontuação abaixo da média, e 1.127 (56,40\%) sujeitos apresentaram-se acima da média, ou seja, uma parcela significativa 
da amostra estudada apresenta dificuldades em ser resiliente.

No que se refere às pontuações dos fatores da Escala de Resiliência, verificou-se, para o Fator 1 - Resolução de ações e valores, uma média de 70,29 $(D P=14,39)$, com pontuação variando entre 14,00 e 98,00. Observou-se que 879 (44,90\%) participantes demonstraram escore abaixo da média, sendo que $1.154(55,10 \%)$ pontuaram acima desta. Esses resultados indicam que a maioria dos participantes é capaz de solucionar ações, levar os planos até o fim e lidar com problemas de alguma forma, sabe enfrentar uma situação de maneiras variadas e pode encontrar uma saída quando se encontra numa situação difícil. Além disso, a maioria desses sujeitos se mostra apta à resolução de valores, conseguindo realizar metas e perceber sentido na sua vida. Deve-se contemplar, todavia, que uma parcela significativa de pessoas (44,90\%) apresenta dificuldades em solucionar problemas.

No que concerne ao Fator 2 - Independência e determinação, a média encontrada foi 26,91 $(D P=5,52)$, com pontuação variando entre 6,00 e 42,00. Observou-se que $894(44,00 \%)$ participantes apresentaram escore abaixo da média, e $1.139(56,00 \%)$ pontuaram acima desta. Diante desses resultados, é possível perceber que uma quantidade satisfatória de pessoas apresenta independência e determinação, mostrando-se frequentemente interessada nos diversos âmbitos da sua vida e capaz de se sentir bem, mesmo quando alguém não gosta dela.

Em relação ao Fator 3 - Autoconfiança e capacidade de adaptação às situações, a média indicada foi uma pontuação de $25,71(D P=4,99)$, com variação de pontuação entre 5,00 e 35,00. Observou-se que 898 $(44,20 \%)$ participantes assinalaram abaixo da média, e 1.135 (55,80\%) apresentaram pontuações acima disso. Pode-se perceber que a maioria dos sujeitos possui autoconfiança e é capaz de adaptar-se às situações. Ou seja, as pessoas que se apresentam acima dessa média, geralmente, são compreendidas como aquelas que conseguem lidar com várias situações ao mesmo tempo, que se sentem aptas a enfrentar os problemas, pois acreditam em si mesmas e possuem o discernimento de não insistir em situações em que não podem fazer nada.

É possível verificar ainda que, nas atribuições das pontuações de resiliência, houve diferenças percebidas pelas comparações realizadas a partir de algumas variáveis sociodemográficas. Não foram significativas, contudo, as diferenças das comparações entre sexos, entre pessoas adotadas ou não, entre os que têm tatuagem ou não- e entre os que já mudaram ou não de emprego por inadaptação, por isso, não serão elencadas abaixo. A seguir, serão registradas apenas as avaliações que se destacaram pela existência de diferenças estatisticamente significativas.

Verificaram-se diferenças estatisticamente significativas nas comparações das pontuações por estado civil, religião, escolaridade, renda e ocupação profissional em todos os fatores. Os divorciados e casados apresentaram mais resiliência do que os solteiros sem relacionamento ou com relacionamento (ver Tabela 3).

Os católicos foram os que apresentaram maior resiliência, e os agnósticos, os que tiveram menor resiliência, enquanto os participantes de várias outras religiões ficaram com pontuações intermediárias (ver Tabela 4).

Os que possuem ensino superior com pós-graduação apresentaram maiores pontuações de resiliência, e os que possuem fundamental incompleto apresentaram pontuações mais baixas, enquanto os participantes com outros níveis de escolaridade ficaram com pontuações intermediárias (ver Tabela 5).

Também se constataram diferenças estatisticamente significativas nas comparações das pontuações por renda, sendo a resiliência crescente conforme a renda (ver Tabela 6).

Já os desempregados são os que possuem menor resiliência, e os aposentados, os que possuem maior resiliência (ver Tabela 7).

Verificou-se menor resiliência apenas no fator 3 entre os que possuem piercing; nos fatores 1 e 3 , entre os que fumam; no fator 2 , entre os que nunca repetiram séries colegiais. Apresentaram menor resiliência, também no fator 1 , os que já repetiram disciplinas na faculdade e os que nunca mudaram de cidade (Ver Tabela 8).

Observaram-se também diferenças estatisticamente significativas nas comparações das pontuações por uso de medicação psíquica. Os que nunca tomaram medicação são os que possuem maior resiliência (Ver Tabela 9).

\section{Discussão}

Considerando-se o índice de Resiliência Total verificado na presente pesquisa $(M=124,60 ; D P=$ $22,69)$ e comparando-o a outros achados na literatura, pôde-se perceber que o resultado ficou abaixo daquele encontrado dentre os participantes do Programa Jovem Aprendiz $(M=133,00 ; D P=31,00)$ (Oliveira \& Godoy, 
Tabela 3.

Comparação das Pontuações de Resiliência por Estado Civil

\begin{tabular}{lccc}
\hline \multicolumn{1}{c}{ Variável } & & \multicolumn{2}{c}{ Distribuição } \\
\cline { 3 - 3 } & & 137,13 & 20,92 \\
Comparação da pontuação de resiliência total & Divorciados & 129,92 & 21,66 \\
por estado civil $(H=48,39, p<0,05)$ & Casados & 124,07 & 22,16 \\
& Em relacionamento & 121,89 & 22,83 \\
\hline & Solteiros & 77,47 & 13,06 \\
Comparação da pontuação do fator 1 por & Divorciados & 73,65 & 13,89 \\
estado civil $(H=47,26, p<0,05)$ & Casados & 70,15 & 14,15 \\
& Em relacionamento & 14,32 \\
\hline & Solteiros & 68,61 & 5,05 \\
Comparação da pontuação do fator 2 por & Divorciados & 29,87 & 5,10 \\
estado civil $(H=41,25, p<0,05)$ & Casados & 27,99 & 15,54 \\
& Em relacionamento & 26,56 & 5,57 \\
\hline & Solteiros & 26,34 & 4,96 \\
Comparação da pontuação do fator 3 por & Divorciados & 27,82 & 4,83 \\
estado civil $(H=37,33, p<0,05)$ & Casados & 26,77 & 4,76 \\
& Em relacionamento & 25,73 & 5,05 \\
\hline
\end{tabular}

Tabela 4.

Comparação das Pontuações de Resiliência por Religião

\begin{tabular}{lccc}
\hline \multicolumn{1}{c}{ Variável } & & \multicolumn{2}{c}{ Distribuição } \\
\cline { 3 - 4 } Comparação da pontuação de resiliência total & Católicos & 128,14 & $D P$ \\
por religião $(H=49,58, p<0,05)$ & Agnósticos & 117,45 & 21,06 \\
\hline Comparação da pontuação do fator 1 por & Católicos & 72,74 & 23,69 \\
estado civil $(H=56,33, p<0,05)$ & Agnósticos & 66,15 & 13,24 \\
\hline Comparação da pontuação do fator 2 por & Católicos & 7,44 & 15,08 \\
estado civil $(H=37,33, p<0,05)$ & Agnósticos & 25,71 & 5,42 \\
\hline Comparação da pontuação do fator 3 por & Católicos & 6,41 & 5,01 \\
estado civil $(H=33,94, p<0,05)$ & Agnósticos & 24,65 & 4,55 \\
\hline
\end{tabular}

2015), pacientes com diabetes $(M=132,50 ; D P=26,32)$ (Motta \& Rosa, 2016), pacientes que sobreviveram ao câncer $(M=141,20 ; D P=8,20)$ (Andrade, 2011) e próximo ao de jovens em situação de vulnerabilidade social $(M=122,52 ; D P=21,85)$ (Haack et al., 2012). Tal dado pode ser um indicativo de que, quanto mais diversa a população observada, maior será a diferença do nível de resiliência (Coimbra \& Morais, 2015).

Nas comparações dos índices de resiliência por dados sociodemográficos, ponderando-se o estado civil, é possível inferir que divorciados e casados apresentam maiores índices quanto à tendência de um sujeito ser 
Tabela 5.

Comparação das Pontuações de Resiliência por Escolaridade

\begin{tabular}{|c|c|c|c|}
\hline \multirow{2}{*}{\multicolumn{2}{|c|}{ Variável }} & \multicolumn{2}{|c|}{ Distribuição } \\
\hline & & $M$ & $D P$ \\
\hline \multirow{2}{*}{$\begin{array}{l}\text { Comparação da pontuação de resiliência total } \\
\text { por escolaridade }(H=68,06, p<0,05)\end{array}$} & $\begin{array}{l}\text { Ensino superior } \\
\text { com pós-graduação }\end{array}$ & 131,33 & 21,03 \\
\hline & $\begin{array}{c}\text { Ensino fundamental } \\
\text { incompleto }\end{array}$ & 110,75 & 36,67 \\
\hline \multirow{2}{*}{$\begin{array}{l}\text { Comparação da pontuação do fator } 1 \text { por } \\
\text { escolaridade }(H=66,70, p<0,05)\end{array}$} & $\begin{array}{l}\text { Ensino superior } \\
\text { com pós-graduação }\end{array}$ & 74,52 & 13,22 \\
\hline & $\begin{array}{c}\text { Ensino fundamental } \\
\text { incompleto }\end{array}$ & 61,42 & 22,09 \\
\hline \multirow{2}{*}{$\begin{array}{l}\text { Comparação da pontuação do fator } 2 \text { por } \\
\text { escolaridade }(H=40,29, p<0,05)\end{array}$} & $\begin{array}{l}\text { Ensino superior } \\
\text { com pós-graduação }\end{array}$ & 28,21 & 5,29 \\
\hline & $\begin{array}{l}\text { Ensino fundamental } \\
\text { incompleto }\end{array}$ & 23,75 & 8,37 \\
\hline \multirow{2}{*}{$\begin{array}{l}\text { Comparação da pontuação do fator } 3 \text { por } \\
\text { escolaridade }(H=64,39, p<0,05)\end{array}$} & $\begin{array}{l}\text { Ensino superior } \\
\text { com pós-graduação }\end{array}$ & 27,06 & 4,64 \\
\hline & $\begin{array}{l}\text { Ensino fundamental } \\
\text { incompleto }\end{array}$ & 23,08 & 8,07 \\
\hline
\end{tabular}

Tabela 6.

Comparação das Pontuações de Resiliência por Renda

\begin{tabular}{llcc}
\hline \multicolumn{1}{c}{ Variável } & & \multicolumn{2}{c}{ Distribuição } \\
\cline { 3 - 4 } Comparação da pontuação de resiliência total & Menor renda & 120,20 & $D P$ \\
por renda $(U=97,69, p<0,05)$ & Maior renda & 141,06 & 23,34 \\
\hline Comparação da pontuação do fator 1 por & Menor renda & 67,67 & 17,19 \\
renda $(U=80,72, p<0,05)$ & Maior renda & 79,75 & 14,89 \\
\hline Comparação da pontuação do fator 2 por & Menor renda & 25,78 & 11,94 \\
renda $(U=94,02, p<0,05)$ & Maior renda & 31,00 & 5,63 \\
\hline Comparação da pontuação do fator 3 por & Menor renda & 24,96 & 5,16 \\
renda $(U=80,68, p<0,05)$ & Maior renda & 29,19 & 5,12 \\
\hline
\end{tabular}

resiliente. Tal fato pode estar relacionado, por exemplo, à presença de um parceiro ao lado do indivíduo ou mesmo à sua condição de estar casado e as particularidades que emergem em ambos os casos. Dentre elas, estão o compartilhamento de responsabilidades e o aumento da rede de suporte social, os quais fazem com que os indivíduos aprendam a lidar com novas situações e a enfrentar novas necessidades. Além disso, verifica-se que as pessoas casadas tendem a reorganizar suas vidas para poder conviver com o outro, tal qual os divorciados, que precisam se readequar à condição de estar sem aquela pessoa com quem viveram por determinado tempo (Carter \& McGoldrick, 1995).

Já no que se refere à religiosidade/espiritualidade, os índices apresentados nesta pesquisa corroboram com os dados da literatura (Melo et al., 2015; Mosqueiro, 2015; Soratto et al., 2016) que afirmam que os níveis de resiliência podem variar de acordo com essas 
Tabela 7.

Comparação das Pontuações de Resiliência por Ocupação Profissional

\begin{tabular}{lccc}
\hline \multicolumn{1}{c}{ Variável } & & \multicolumn{2}{c}{ Distribuição } \\
\cline { 3 - 4 } Comparação da pontuação de resiliência total & Desempregado & 118,01 & $D P$ \\
por ocupação $(H=110,96, p<0,05)$ & Empregado & 128,86 & 23,37 \\
& Aposentado & 132,47 & 22,23 \\
Comparação da pontuação do fator 1 por & Desempregado & 66,26 & 24,69 \\
ocupação $(H=94,02, p<0,05)$ & Empregado & 72,59 & 14,92 \\
& Aposentado & 75,59 & 13,70 \\
Comparação da pontuação do fator 2 por & Desempregado & 25,62 & 16,33 \\
ocupação $(H=99,60, p<0,05)$ & Empregado & 27,99 & 5,62 \\
\hline \multirow{2}{*}{ Comparação da pontuação do fator 3 por } & Aposentado & 28,82 & 5,13 \\
ocupação $(H=107,10, p<0,05)$ & Desempregado & 24,30 & 4,90 \\
\hline
\end{tabular}

Tabela 8.

Comparação das Pontuações de Resiliência por Uso de Piercing, Fumo, Escolaridade e Mudança de Cidade

\begin{tabular}{|c|c|c|c|}
\hline \multirow{2}{*}{\multicolumn{2}{|c|}{ Variável }} & \multicolumn{2}{|c|}{ Distribuição } \\
\hline & & $M$ & $D P$ \\
\hline \multirow{2}{*}{$\begin{array}{l}\text { Comparação da pontuação do fator } 3 \text { por uso } \\
\text { de piercing }(U=2,70, p<0,05)\end{array}$} & Possuem piercing & 24,90 & 4,53 \\
\hline & Não possuem & 25,77 & 5,02 \\
\hline \multirow{2}{*}{$\begin{array}{l}\text { Comparação da pontuação do fator } 1 \text { por uso } \\
\text { de fumo }(U=2,42, p<0,05)\end{array}$} & Fumante & 67,56 & 15,11 \\
\hline & Não fumante & 70,53 & 14,30 \\
\hline \multirow{2}{*}{$\begin{array}{l}\text { Comparação da pontuação do fator } 3 \text { por uso } \\
\text { de fumo }(U=2,41, p<0,05)\end{array}$} & Fumante & 24,66 & 5,63 \\
\hline & Não fumante & 25,80 & 4,92 \\
\hline \multirow{2}{*}{$\begin{array}{l}\text { Comparação da pontuação do fator } 2 \text { entre } \\
\text { os que repetiram ou não series colegiais }(U= \\
2,61, p<0,05)\end{array}$} & Nunca repetiram & 26,79 & 5,48 \\
\hline & Já repetiram & 27,49 & 5,65 \\
\hline \multirow{2}{*}{$\begin{array}{l}\text { Comparação da pontuação do fator } 1 \text { entre } \\
\text { os que repetiram ou não de disciplinas } \\
\text { acadêmicas }(U=2,52, p<0,05)\end{array}$} & Nunca repetiram & 75,05 & 14,02 \\
\hline & Já repetiram & 69,21 & 14,84 \\
\hline \multirow{2}{*}{$\begin{array}{l}\text { Comparação da pontuação do fator } 1 \text { entre os } \\
\text { que já mudaram ou não de cidade }(U=2,33 \text {, } \\
p<0,05)\end{array}$} & Nunca mudaram & 26,77 & 5,46 \\
\hline & Já mudaram & 27,44 & 5,70 \\
\hline
\end{tabular}

características. Por exemplo, observa-se que a religiosidade está associada à maior resiliência em sujeitos com depressão em internação psiquiátrica (Mosqueiro, 2015) e em pacientes oncológicos (Soratto et al., 2016). Nessa perspectiva, pode-se inferir que o fato de ser vinculado a uma religião pode contribuir no sentido que se dá a vida e na construção de mecanismos de defesa para o enfrentamento das dificuldades, obtendo-se maior apoio e segurança nos momentos de adversidades. Além disso, pode-se pensar que, para o agnóstico, o nível de 
Tabela 9.

Comparação das Pontuações de Resiliência por Uso de Medicação Psiquiátrica

\begin{tabular}{|c|c|c|c|}
\hline \multirow{2}{*}{\multicolumn{2}{|c|}{ Variável }} & \multicolumn{2}{|c|}{ Distribuição } \\
\hline & & \multirow{2}{*}{\begin{tabular}{c|}
$M$ \\
119,30
\end{tabular}} & \multirow{2}{*}{$\frac{D P}{24,47}$} \\
\hline $\begin{array}{l}\text { Comparação da pontuação } \\
\text { de resiliência total por uso de }\end{array}$ & $\begin{array}{l}\text { Nunca fez uso de } \\
\text { medicamento }\end{array}$ & & \\
\hline medicação psiquiátrica & Já fez uso de medicamento & 114,12 & 23,68 \\
\hline$(H=7,73, p<0,05)$ & Faz uso de medicamento & 114,12 & 23,68 \\
\hline \multirow{3}{*}{$\begin{array}{l}\text { Comparação da pontuação do } \\
\text { fator } 1 \text { por uso de medicação } \\
\text { psiquiátrica } \\
(H=7,78, p<0,05)\end{array}$} & $\begin{array}{l}\text { Nunca fez uso de } \\
\text { medicamento }\end{array}$ & 66,68 & 15,53 \\
\hline & Já fez uso de medicamento & 63,90 & 15,11 \\
\hline & Faz uso de medicamento & 63,90 & 15,11 \\
\hline \multirow{3}{*}{$\begin{array}{l}\text { Comparação da pontuação do } \\
\text { fator } 2 \text { por uso de medicação } \\
\text { psiquiátrica } \\
(H=5,48, p<0,05)\end{array}$} & $\begin{array}{l}\text { Nunca fez uso de } \\
\text { medicamento }\end{array}$ & 26,13 & 15,74 \\
\hline & Já fez uso de medicamento & 24,95 & 5,63 \\
\hline & Faz uso de medicamento & 24,95 & 5,63 \\
\hline \multirow{3}{*}{$\begin{array}{l}\text { Comparação da pontuação do } \\
\text { fator } 3 \text { por uso de medicação } \\
\text { psiquiátrica }(H=6,47, p<0,05)\end{array}$} & $\begin{array}{l}\text { Nunca fez uso de } \\
\text { medicamento }\end{array}$ & 24,89 & 5,28 \\
\hline & Já fez uso de medicamento & 23,48 & 5,37 \\
\hline & Faz uso de medicamento & 23,48 & 5,37 \\
\hline
\end{tabular}

tendência à resiliência seja minimizado, uma vez que ele não apresenta a segurança ou crença necessária de que algum recurso da ordem espiritual poderá contribuir para combater seus problemas (Melo et al., 2015).

Pode-se constatar que a maior tendência à resiliência pode estar associada ao nível de escolaridade. Essa circunstância pode ser entendida pelo fato de as pessoas terem adquirido, além do conhecimento sobre suas áreas, maneiras de organizar suas vidas, cumprir metas e prazos, saber lidar com as situações de serem testadas e, com isso, obterem aprovações e reprovações. Ademais, podem obter experiências em fechar ciclos e começar outros novos, vivenciando, frequentemente, novas situações e desafios. Além disso, reconhece-se a influência do planejamento e da ressignificação de alguns acontecimentos sobre a resiliência. Essas inferências também podem ser encontradas nos estudos de Lopes e Massinelli (2013).

Em relação ao tipo de ocupação, infere-se que aqueles que estão aposentados apresentam maior resiliência em relação aos indivíduos sem ocupação. Uma hipótese para esse resultado é que as situações apresentadas durante o tempo de serviço podem tê-los ajudado a desenvolver habilidades de enfrentamento. $\mathrm{Na}$ situação do desemprego, pode-se depreender que, diante das respostas negativas frente à procura de um trabalho, a pessoa que se encontra desesperançosa pode desistir das tentativas de conseguir um emprego, não favorecendo a visualização de outras possibilidades para superar essa condição. De acordo com o Ministério da Previdência Social (MPS, 2008), apesar de ocorrer uma exclusão do aposentado do mercado de trabalho, há benefício diante da diminuição do estresse devido ao domínio da sua situação financeira, o desenvolvimento de liberdade na ausência de uma subordinação ao emprego e o apoio fraterno. Em outras palavras, fatores que possibilitam a conservação do bem-estar e elaboração de planos para o futuro (Castanheira, 2013).

Além da ocupação laboral, percebe-se a relação da renda com os níveis de resiliência, da qual se infere que pessoas com maior renda apresentam maior propensão a serem mais resilientes em relação às pessoas com menor renda, devido às melhores condições de vida. A partir disso, entende-se que aqueles com maior renda, ao passarem por alguma situação adversa, costumam ter maior habilidade para retornar ao equilibrio.

Conforme Sapienza e Pedromônico (2005), é possível associar fatores de risco à resiliência. Quando esses aspectos de ameaça são associados a outras adversidades, eleva-se a possibilidade de um resultado 
desfavorável para o desenvolvimento do sujeito. Assim, estar em situação financeira adversa dificulta a emissão de respostas satisfatórias e o desenvolvimento de habilidades. Por exemplo, quando a pessoa é exposta a uma realidade em que seu ganho financeiro só pode ser destinado para seu sustento, pode ocorrer o desinvestimento em fatores que contribuem para seu bem-estar físico e psíquico.

No que tange ao uso de piercing, pode-se inferir que os indivíduos que o utilizam são inseguros na busca de independência, evidenciando maiores dificuldades em lidar com contrariedades. Para Teixeira (2012), as práticas de autonomia da adolescência baseadas em passeios noturnos, uso de piercings e tatuagens, entre outros rituais, podem atingir comportamentos perigosos, apresentando sentimentos voláteis que vão de encontro aos fatores de proteção, isto é, elementos que contribuem para a superação de situações de risco e conservação de um crescimento satisfatório.

A análise dos dados também permitiu observar que os indivíduos que fumam tendem a ser mais incapazes de lidar com as adversidades. Isso pode ocorrer porque parte dos fumantes são pessoas que possuem previamente dificuldade em lidar com dificuldades e utilizem o fumo como forma de suprimir ou se refugiar de angústias provocadas por situações aversivas, como conflitos familiares ou de trabalho (Fortes et al., 2009).

Outro fator relevante depreende-se em relação aos que já repetiram séries colegiais e os que não repetiram, salientando-se aqui o fator 2 , que avalia independência e determinação. Como visto, os que nunca repetiram apresentaram menores pontuações de resiliência do que os que já repetiram. A partir disso, sugere-se como hipótese que a maior resiliência de pessoas que já repetiram séries colegiais nesse fator pode ocorrer pelo fato de esses indivíduos não desejarem passar por essa situação novamente, logo se sentindo determinados para agir de forma mais eficaz e independente (Duarte, 2016).

Diante do fator 1 e 3, que avaliam a resolução de ações e valores e a capacidade de autoconfiança e adaptação às situações, comparando-se os índices de resiliência das pessoas que já desistiram do curso e das que não desistiram, constata-se que os sujeitos que nunca desistiram do curso apresentam maiores índices quanto à tendência de um sujeito ser resiliente. Compreende-se que tal fato ocorre por apresentarem mais determinação e assertividade na escolha do curso e por não passarem pelos momentos de indecisão e de desistência, tendo já dado início a um curso universitário (Oliveira \& Godoy, 2015).
Sobre esse aspecto, Cookson (1990) destaca que a evasão de um curso pode ser entendida como um fenômeno causado pela combinação de características dos alunos e de suas circunstâncias de vida. Existem comportamentos que podem contribuir para tal ato, tais como a falta de motivação, fase do estágio do desenvolvimento adulto, as pressões emergentes de trabalho, das atividades do curso e das possíveis variações de sua própria personalidade. Diante de tais barreiras e limitações, é possível que alunos com menor capacidade de resolução de ações e valores (Fator 1), ou seja que possuem dificuldade de levar planos até o fim, lidar com problemas e motivados consigo e com o que fazem, e menor autoconfiança e capacidade de adaptação às situações (Fator 3), ou seja, com independência na solução de seus problemas, tendam a desistir do curso.

Todavia, o Fator 2 mostrou que aqueles que já desistiram apresentaram maior resiliência em relação àqueles que nunca desistiram. Aqui observam-se melhores pontuações nos itens que abordam o fato de cuidar de si sozinho e sentir-se bem consigo, ainda que haja pessoas que não gostam de você. Acredita-se que o fato de esses indivíduos terem adquirido uma maior resistência e motivação ao desistir de algo resulta no início de um novo ciclo, buscando meios e artifícios para driblar e superar tal adversidade (Silva et al., 2014).

A análise dos dados gerais desta pesquisa proporcionou compreender mais a respeito sobre os níveis de resiliência entre as pessoas que já repetiram disciplinas na faculdade e aquelas que não o fizeram. Conforme exposto nos resultados, pode-se constatar que a reprovação em disciplinas na faculdade é um fator bastante significativo para os estudantes na graduação. Percebe-se que os alunos que nunca repetiram nenhuma cadeira apresentam maior propensão a serem mais resilientes em relação àqueles que já repetiram alguma. Associa-se isso ao fato de os alunos que passaram pela experiência de repetir a mesma disciplina sentirem-se desmotivados e desestimulados, uma vez que já cursaram e se depararam com o conteúdo e a experiência que a disciplina oferece mais de uma vez (Duarte, 2016).

Diante dos resultados, também pode ser observado o fato de que se ter morado em mais de um local aumenta a capacidade de resiliência em relação a indivíduos que nunca mudaram de cidade. À medida que as pessoas se submetem a sair do seu local de moradia fixa e experimentam iniciar um novo ciclo de vida (casa nova, vizinhos novos), estão se sujeitando a vivenciar experiência positivas e negativas. Além disso, surge a possibilidade de se criarem novos laços 
sociais, podendo-se obter maior apoio e segurança nos momentos de adversidades, fortalecendo, assim, suas capacidades de superação em reverter e enfrentar situações aversivas. Em oposição, pessoas que nunca se mudaram não se submeteram ou se expuseram poucas vezes a momentos que exigissem motivação e preparo para superar grandes adversidades, não estimulando mecanismos de defesa para o enfrentamento das dificuldades (Sapienza \& Pedromônico, 2005).

Por fim, considerando o uso de medicações psiquiátricas, pode-se constatar que o fato de se usar medicação faz com que o nível de tendência à resiliência seja minimizado, em contraposição aos indivíduos que não utilizam desse tipo de medicação. Observou-se que os indivíduos reféns da medicação se sentem mais vulneráveis e menos capazes para lidar com situações adversas. Isso pode ocorrer devido à dependência que a droga pode vir a causar como efeito, além da própria doença mental em si, podendo provocar delírios e alucinações (Oliveira et al., 2014).

\section{Considerações Finais}

Neste estudo, foi proposto verificar se as características sociodemográficos podem influenciar no nível de resiliência de uma amostra da população brasileira. Partindo-se do pressuposto de que a resiliência é uma capacidade ou habilidade de poder adequar-se às circunstâncias de crise, foi possível constatar em que âmbito determinada característica do sujeito está relacionada à sua maior ou menor tendência a ser resiliente.

Por meio da Escala de Resiliência desenvolvida por Wagnild e Young (1993), verificou-se a pontuação geral dos três fatores citados (Resolução de ações e valores, Independência e determinação e Autoconfiança e capacidade de adaptação às situações) e constatou-se, primeiramente, que uma parcela significativa da amostra estudada apresenta dificuldades em ser resiliente. As características que parecem contribuir para um maior nível de resiliência estão entre as pessoas divorciadas ou casadas, católicas, com ensino superior com pós-graduação, aposentadas, que possuem maior renda, não possuem piercing, não são fumantes, repetiram alguma série no colegial, não desistiram do curso universitário, já desistiram do curso universitário, não repetiram disciplinas na faculdade, já mudaram de cidade por motivo de inadaptação e não fazem uso de medicação psíquica.

Ressalta-se que possuir essas características não garante maior resiliência- e que essas não são condições determinantes. Pondera-se, pois, que existe uma predisposição entre esses sujeitos para uma maior resiliência, seja devido à variável; ou por outra característica comum a esses grupos. Informações que são primordiais para compreender fatores que contribuem, ou não, predispõem, ou não, a resiliência da população.

Pode-se considerar como aspecto limitante deste estudo o fato de ter sido pontual, não sendo possível realizar uma pesquisa a longo prazo, de forma longitudinal, na busca de compreender fatores individuais e ambientais correlacionados à mudança da resiliência. Desse modo, entende-se que os resultados deste estudo poderão contribuir para uma melhor compreensão acerca das características de uma população que torna maior ou menor a propensão a ser resiliente. $\mathrm{O}$ intuito envolve a contribuição para novas estratégias de assistência no trabalho em saúde, assim como favorecer o ensino e a pesquisa para a construção de novas concepções sobre o tema e para a consolidação acerca da importância sobre se tornar resiliente.

\section{Referências}

Andrade, F. P., Muniz, R. M., Lange, C., Schwartz, E., \& Echevarria, M. E. (2013). Perfil sociodemográfico e econômico dos sobreviventes ao câncer segundo o grau de resiliência. Texto contexto - enfermagem, 22(2), 476-484. doi: 10.1590/S0104-07072013000200025

Ministério da Previdência Social. (2008). Envelhecimento e dependência: Desafios para a Organižação da Proteção Social. Brasília: MPS.

Carter. B., \& McGoldrick, M. (1995). As mudanças no ciclo de vida familiar. Porto Alegre: Artes Médicas.

Castanheira, F. P. D. (2013). A relação entre a resiliência e a vulnerabilidade ao stresse: estudo numa organização de práticas positivas (Dissertação de mestrado não publicada). Instituto Superior de Línguas e Administração, Coimbra, Portugal.

Coimbra R. M., \& Morais N. A. (2015). A resiliência em questão: Perspectivas teóricas, pesquisa e intervenção. Porto Alegre: Artmed.

Cookson, P. (1990). Persistence in distance education. Em M. G. Moore, A. B. Quigley, P. Cookson \& J. Donaldson. Contemporary Issues in American Distance Education. Oxford: Pergamon Press.

Duarte, R. C. A. (2016). O efeito da reprovação na motivação dos alunos (Dissertação de mestrado não publicada). Instituto Politécnico do Porto, Porto, Portugal. 
Favero, L. P., \& Belfiore, P. (2017). Manual de análise de dados: Estatística e modelagem multivariada com Excel®, SPSS E STATA®. São Paulo: Elsevier.

Ferreira, F. G. B. (2013). Análise do Programa Bolsa Família quanto à resiliência familiar no município de Sumé-PB (Trabalho de conclusão de curso não publicado). Universidade Estadual da Paraíba, Campina Grande, Paraíba.

Fontes, A. P., Fattori, A., D’Elboux, M. J., \& Guariento, M. E. (2015). Resiliência psicológica: Fator de proteção para idosos no contexto ambulatorial. Revista Brasileira de Geriatria e Gerontologia, 18(1), 7-17. doi: 10.1590/1809-9823.2015.13201

Fortes, T. F. R., Portuguez M. W., \& Argimon I. I. L. (2009). A resiliência em idosos e sua relação com variáveis sociodemográficas e funções cognitivas. Estudos de Psicologia, 26(4), 455-463. doi: 10.1590/ S0103-166X2009000400006

Haack, K. R., Vasconcellos, J. S. L., Pinheiro, S. D., \& Prati, L. E. (2012). Resiliência em adolescentes em situação de vulnerabilidade social. Revista Interinstitucional de Psicologia, 5(2), 1-12. Recuperado de http://pepsic.bvsalud.org/pdf/gerais/v5n2/ v5n2a07.pdf

Laranjeira, J. C. (2007). Do vulnerável ser ao resiliente envelhecer: Revisão da literatura. Psicologia: Teoria e Pesquisa, 23(3), 327-332. doi: 10.1590/ S0102-37722007000300012

Lopes, S. R. A., \& Massinelli, C. J. (2013). Perfil e nível de resiliência dos cuidadores informais de idosos com Alzheimer. Aletheia, 40, 134-145. Recuperado de http://pepsic.bvsalud.org/scielo.php?script $=$ sci_arttext\&pid=S141303942013000100012\&ln$\mathrm{g}=\mathrm{pt} \& \mathrm{t} \operatorname{lng}=\mathrm{pt}$

McCann, C., Beddoe, E., McCormick, K., Huggarg, P., Kedge, S., Adamson, C. \& Huggard, J. (2013). Resilience in the health professions: A review of recent literature. International Journal of Wellbeing, 3(1), 60-81. doi: 10.5502/ijw.v3i1.4

Melo, C. F., Sampaio, I. S., Souza, D. L. A., \& Pinto, N. S. (2015). Correlação entre religiosidade, espiritualidade e qualidade de vida: Uma revisão de literatura. Estudos e Pesquisas em Psicologia (On-line), 15(2), 447-464. Recuperado de http://pepsic. bvsalud.org/scielo.php?script $=$ sci_arttext\&pi$\mathrm{d}=$ S1808-42812015000200002\&lng $=$ pt\&tlng $=$ pt
Mosqueiro, B. P. (2015). Religiosidade, resiliência e depressão em pacientes internados (Dissertação de mestrado não publicada), Universidade Federal do Rio Grande do Sul, Porto Alegre, Rio Grande do Sul.

Motta, B. F. B., \& Rosa, J. H. S. (2016). Aspectos sociais da resiliência em pacientes com diabetes mellitus tipo II. Revista Cientifica Fagoc Saúde, 1(1), 27-36. Recuperado de http://revista.fagoc.br/index.php/ saude/article/view/24

Munist, M., Santos, H., Kotliarenco, M. A., Ojeda, E. N. S., Infante, F., \& Grotberg, E. (1998). Manual de identificación e promoción de la resiliencia. Washington: Organización Panamericana de la salud.

Oliveira, R. M., Siqueira, A. C., Santos, J. L. F. \& Furegato, A. R. F. (2014). Nicotine dependence in the mental disorders, relationship with clinical indicators, and the meaning for the user. Revista Latino-Americana de Enfermagem [online], 22(4), 685692. doi: 10.1590/0104-1169.3549.2468

Oliveira, A. L., \& Godoy, M. M. C. (2015). O processo de resiliência do jovem aprendiz e as estratégias de conciliação estudo-trabalho. Boletim de Psicologia, 65(143), 175-191. Recuperado de http://pepsic. bvsalud.org/scielo.php?script $=$ sci $\quad$ arttext\&pi$\mathrm{d}=$ S0006-59432015000200006\&lng=pt\&tlng=pt

Pesce, R. P., Assis, S. G., Avanci, J. Q., Santos, N. C., Malaquias, J. V., \& Carvalhaes, R. (2005). Adaptação transcultural, confiabilidade e validade da escala de resiliência. Cadernos de Saúde Pública, 21(2), 436-448. doi: 10.1590/S0102-311X2005000200010.

Silva, J. S., Pinto, F. R., Nogueira, T. V., \& Ferreira, T. C. (2014). Resiliência em discentes de administração, por idade, religiosidade e gênero. Revista da Faculdade de Administração e Economia, 5(2), 1-22. doi: 10.15603/2176-9583/refae.v5n2p141-162

Poletto, M., Wagner, T. M. C., \& Koller, S. H. (2004). Resiliência e desenvolvimento infantil de crianças que cuidam de crianças: Uma visão em perspectiva. Psicologia: Teoria e Pesquisa, 20(3), 241-250. doi: 10.1590/S0102-37722004000300005

Ripar, A. A., Evangelista, F. S. F. Q., \& Paula, F. V. (2008). Resiliência e gênero. Psicólogo informação, 12(12), 33-52. Recuperado de http://pepsic. bvsalud.org/scielo.php?script=sci_arttext\&pi$\mathrm{d}=$ S1415-88092008000100003

Rutter M. (1985). Resilience in the face of adversity: Protective factors and resistance to psychiatric 
disorder. British Journal of Psychiatry, 22(1), 323-356. doi: 10.1192/bjp.147.6.598

Sapienza, G., \& Pedromônico, M. R. M. (2005). Risco, proteção e resiliência no desenvolvimento da criança e do adolescente. Psicologia em Estudo, 10(2), 209-216. doi: 10.1590/S1413-73722005000200007

Soratto, M. T., Silva, D. M., Zugno, P. I., \& Daniel, R. (2016). Espiritualidade e resiliência em pacientes oncológicos. Revista Saúde e Pesquisa, 9(1), 53-63. doi: 10.177651/1983-1870.2016v9n1p53-63

Sousa, V. F. S., \& Araújo, T. C. C. F. (2015). Estresse ocupacional e resiliência entre profissionais de saúde. Psicologia: Ciência e Profissão, 35(3), 900-915. doi: 10.1590/1982-370300452014

Teixeira, A. (2012). A adolescência e a juventude como culturas. Theologica, 47(1), 99-113. Recuperado de http://hdl.handle.net/10400.14/13378
Wagnild G. M., \& Young H. M. (1993). Development and psychometric evaluation of resilience scale. $J$ Nurs Meas, 1(2), 165-78. Recuperado de https:// www.ncbi.nlm.nih.gov/pubmed/7850498

Werner E. E, \& Smith R. S. (1982). Vulnerable but invincible: A longitudinal study of resilient children and youth. New York: Mcgraw-Hill.

Werner E. E, \& Smith R. S. (1992). Overcoming the odds: High risk children from birth to adulthood. Ithaca: Cornell University Press.

Werner E. E, \& Smith, R. S. (1977). Kauai's children come of age. Honolulu: University of Hawaii Press.

Recebido em: 29/03/2017 Reformulado em: 17/10/2018, $16 / 01 / 2019,13 / 02 / 2019$ Aceito: 24/03/2019 
Sobre os autores:

Cynthia de Freitas Melo é doutora em Psicologia (UFRN), professora do Programa de Pós-Graduação em Psicologia da Universidade de Fortaleza (UNIFOR) e coordenadora do Laboratório de Estudos e Práticas em Psicologia e Saúde (LEPP-Saúde).

ORCID: https://orcid.org/0000-0003-3162-7300

E-mail:cf.melo@yahoo.com.br

José Eurico de Vasconcelos Filho é doutor em informática (PUC-Rio), professor de Engenharia da Computação e do Mestrado em Inovação Tecnológica em Enfermagem da Universidade de Fortaleza e coordenador do Laboratório de Inovação em TIC - NATI.

ORCID: https://orcid.org/0000-0002-6881-0814

E-mail: euricovasconcelos@uniofr.br

Marina Braga Teófilo é psicóloga (2017) pela Universidade de Fortaleza.

ORCID: https://orcid.org/0000-0003-0965-2972

E-mail: marina.teofilo@yahoo.com.br

Amanda Martins Suliano é psicóloga (2017) pela Universidade de Fortaleza.

ORCID: https://orcid.org/0000-0003-3685-5695

E-mail: manda.mg@hotmail.com

Érika Carolinne Cisne é psicóloga (2017) pela Universidade de Fortaleza.

ORCID: https://orcid.org/0000-0002-8654-8610

E-mail: erikaccisne@gmail.com

Ronaldo Almeida de Freitas Filho é graduado em engenharia da computação (2016) pela Universidade de Fortaleza. ORCID: https://orcid.org/0000-0001-8143-6671

E-mail: ronaldoalm1812@edu.unifor.br

Contato com os autores:

Cynthia de Freitas Melo

Av. Washington Soares, 1321, Bloco E, Sala E-01, Bairro Edson Queiroz

Fortaleza-CE, Brasil

CEP: 60811-341 